\title{
Exakte Kenntnisse der Gesichtsanatomie ein Muss
}

„Wir können Schönheit kreieren, wenn wir auf die Charakteristika Einfluss nehmen, die ein Gesicht schön wirken lassen“, sagte Dalvi Humzah, West Midlands, UK. Laut Humzah entscheiden neben den Proportionen 7 Faktoren darüber, ob ein Gesicht als schön gilt: Haut (Struktur, Pigmentierung, Elastizität, Volumen), Gesichtsform, Distanz der Augen, Stirnhöhe und -kontur sowie Form der Augenbrauen, Nase und Lippen.

Zur Glättung dynamischer Falten wie im Bereich der Glabella bieten sich Injektionen mit Botulinumtoxin Typ A an. Statische Falten können mit Hyaluronfillern (wie Restylane ${ }^{\circledast}$ ) unterspritzt werden. Zudem sind sie für den lokalen Volumenaufbau z. B. an den Lippen sowie zur Gesichtskonturierung geeignet. Wichtig ist die genaue Kenntnis der anatomischen Strukturen wie Blutgefäße, Fettkompar- timente, Ligamente, Muskeln, Nervenund Knochengewebe, sowie das Verständnis, wie sich diese im Laufe der Zeit veränderten. Dem Volumenverlust der Haut könne man gut mit Skinboostern entgegen wirken.

Auch Muskeln und Knochen altern. Am Schädel vergrößere sich die OrbitaÖffnung A-förmig - hier könne eine Rejuvenierung durch Fillerinjektionen entlang des unteren Orbitalrandes erreicht werden. Das Os nasale weiche zurück, so dass die Nase im Alter größer wirke, daher gelte es, die Nasenflügel mit Volumen zu unterstützen. Darüber hinaus senkten sich die Wangenknochen, Kiefer und Zähne wichen zurück, so dass die Lippen flacher wirkten und auch der Unterkieferknochen verliere deutlich an Höhe.

Es sei auch wichtig, das richtige Produkt an der richtigen Stelle einzusetzen.
Er riet für die Lippen speziell geeignete Filler zu verwenden, um unnatürliche Ergebnisse mit „steifen“ Lippen zu vermeiden. In der Stirn- und Glabellaregion sollte Botulinumtoxin oberflächlich injiziert werden, um Supraorbital- und Supratrochleargefäße oder -nerven nicht $\mathrm{zu}$ verletzen und z.B. eine Ptosis zu umgehen. Auch müsse bei Volumenbehandlungen im Schläfenbereich besonders auf Nerven und Blutgefäße (Arteria temporalis superficialis) geachtet werden, da hier eine fehlerhafte Fillerinjektion bis zur Blindheit führen könne, warnte Humzah.

Dr. Gerhard Sattler, Darmstadt, demonstrierte den Einsatz von Botulinumtoxin (Azzalure ${ }^{\circledast}$ mit dem Ziel eine Schonarthrophie der Brauenmuskulatur bei einem Patienten $\mathrm{zu}$ erreichen, der über seine wulstigen Augenbrauen klagte. Sattler riet zur Absenkung des 4. und 5. Injektionspunktes, um so die mediale Immobilisation des M. frontalis mit lateraler Kompensation - den sogenannten „Spock-Effekt" - zu vermeiden. SusannePickl

Galderma Ästhetiktag, Düsseldorf, 30.5.2015; Veranstalter: Galderma Laboratoirum

\section{Eine Pflegelinie adressiert alle Symptome}

Eine schwere Xerosis cutis ist immer mit Juckreiz korreliert, erläuterte Prof. Dr. Sonja Ständer von der Universitätshautklinik Münster. Die Expertin nannte eine Vielzahl intrinsischer Faktoren, die als Ursache für trockene Haut im Alter infrage kommen: verringerte Lipidproduktion im Stratum corneum, schlechtere Barrierefunktion, veränderte $\mathrm{pH}$-Werte und nicht zuletzt ein verlangsamter Zell-Turnover durch veränderte Hormonspiegel. $\mathrm{Zu}$ den extrinsischen Faktoren können die Einnahme von Medikamenten, Pflegegewohnheiten oder Umwelteinflüsse zählen.

Pruritus macht den Betroffenen besonders zu schaffen. Eine wichtige Funktion in der Pruritus-Pathogenese kommt der Dermatologin zufolge dem Protease-aktivierten Rezeptor 2 (PAR-2) zu. Erhöht sich der $\mathrm{pH}$-Wert der Haut in Richtung alkalisch, werden vermehrt Serinproteinasen aktiviert, die an PAR-2 binden. Diese Aktivierung von PAR-2 führt dann zu Juckreiz und Schmerz.
Mit der Haut als „humanem Mikrobiom" beschäftigte sich Prof. Dr. Andreas Wollenberg, LMU München. Bei einem neurodermitischen Schub verändere sich die Zusammensetzung der Keime auf der Haut, ihre Diversifität nehme ab: „Ein Patient mit atopischem Ekzem hat sehr viel mehr Staphylokokken, und zwar Staphylococcus aureus mit pro-entzündlichem Effekt, und nicht Staphylococcus epidermidis. "Aus Untersuchungen wisse man, dass sich mit XeraCalm A.D. die Staphylococcus-aureus-Besiedelung auf der Haut auf niedrigem Niveau halten lasse. Zu verdanken sei das I-modulia ${ }^{\circledast}$, einem Lysat aus dem Keim Aquaphilus dolomiae, der ausschließlich im Thermalwasser von Avène vorkommt. I-modulia inhibiert Wollenberg zufolge die Genexpression zahlreicher proinflammatorischer Zytokine und induziert die Expression antimikrobieller Peptide, die das Wachstum von Keimen reduzieren. „Wir haben also, ohne dass wir einen lebenden Keim auf die Haut bringen müssen, durch diesen Botenstoff aus den Thermalwasserkeimen eine Hemmung der lokalen Entzündungsreaktion.“ Wie Wollenberg weiter ausführte, greife das Lysat auch direkt in den Signalweg für Juckreiz ein, indem es dosisabhängig die Signaltransduktion durch PAR-2 reduziert.

Somit steht mit XeraCalm A.D. von Eau Thermale Avène eine Pflegelinie zur Verfügung, die in ihrer Wirkstoffkombination speziell auf trockene Haut und Neurodermitis abgestimmt ist. Neben bakteriologisch reinem Avène Thermalwasser kommt mit I-modulia ${ }^{\circledR}(0,4 \%)$ ein biotechnologischer Wirkstoff gegen Entzündungen und für eine verbesserte Wundheilung zur Anwendung. Komplettiert wird die Wirkstoffkombination durch Cer-Omega (2,3\%), das neben Ceramiden auch ungesättigte Omega6-Fettsäuren beinhaltet und somit dem Wasserverlust entgegenwirkt und die Barrierefunktion unterstützt. Ursula Hilpert

Mittagsseminar "Thermalwasser: Quelle der Hautberuhigung" bei der 48. Tagung der Deutschen Dermatologischen Gesellschaft am 29.4.2015 in Berlin; Veranstalter: EAU THERMALE Avène 